J. Lake Sci.(湖泊科学), 2009, 21(5): 631-636

http://www.jlakes.org. E-mail: jlakes@niglas.ac.cn

(C2009 by Journal of Lake Sciences

\title{
太湖、洞庭湖野生青虾肌肉中有机氯农药的气相色谱-质谱法测定"
}

\author{
史双昕 ${ }^{1}$, 卢婉云 ${ }^{2}$, 郡丁丁 ${ }^{1}$, 周 丽 $^{1}$, 张 烃 $^{1}$, 黄业茹 ${ }^{1}$ \\ (1: 国家环境分析测试中心，国家环境保护二噁英污染控制重点实验室，北京 100029) \\ (2: 中国人民大学环境学院, 北京 100081)
}

摘 要: 使用气相色谱质谱技术测定了青虾肌肉组织中的 8 种有机氯农药, 包括六氯苯、氯丹、滴滴涕 $\left(\sum \mathrm{DDTs}\right)$ 、艾氏剂、狄 氏剂、异狄氏剂、七氯和灭蚁灵. 对太湖和洞庭湖 15 个点位 29 组野生青虾样品的测定结果显示, 青虾肌肉组织内残留的有机 氯农药主要是六氯苯和滴滴涕, 六氯苯含量为 $n d-13.2 \mu \mathrm{g} / \mathrm{kg}$ (湿重), 滴滴涕含量为 $0.790-5.82 \mu \mathrm{g} / \mathrm{kg}$ (湿重). 洞庭湖青虾肌肉中 有机氯农药的含量略高于太湖青虾, 两湖青虾肌肉残留有机氯农药在枯水期时的含量多数大于丰水期时的含量.

关键词: 气相色谱质谱法; 青虾; 有机氯农药; 太湖; 洞庭湖

\section{Determination of organochlorine pesticides in wild shrimps muscle tissue in Lake Taihu and Lake Dongting by Gas Chromatogrphy/Mass Spectrometry}

\author{
SHI Shuangxin ${ }^{1}$, LU Wanyun ${ }^{2}$, SHAO Dingding ${ }^{1}$, ZHOU Li $^{1}$, ZHANG Ting $^{1} \&$ HUANG Yeru \\ (1: National Research Center for Environmental Analysis and Measurements, Dioxins Laboratory of the National Research Center \\ for Environment Analysis and Measurement, Beijing 100029, P.R.China) \\ (2: Environment College, Renmin University of China, Beijing 100081, P.R.China)
}

Abstract: Twenty-nine shrimp samples from 15 sites in Lake Taihu and Lake Dongting were collected and measured, and 8 kinds of organochlorine pesticides (OCPs) in wild shrimps muscle tissue were analyzed with Gas Chromatography/Mass Spectrometry. Hexachlorobenzene(HCB), Chlordane, $\sum \mathrm{DDTs}\left(p, p^{\prime}\right.$-DDT、 $o, p^{\prime}$-DDT 、 $p, p^{\prime}$-DDE 、 $o, p^{\prime}$-DDE 、 $p, p^{\prime}$-DDD、 $o, p^{\prime}$-DDD), Aldrin, Dieldrin, Endrin, Heptachlor and Mirex were quantified. The results showed HCB and $p, p^{\prime}$-DDE were detected widely in all shrimp samples. $\mathrm{HCB}$ residues in shrimp muscle ranged from nd to $13.2 \mu \mathrm{g} / \mathrm{kg}$ and $p, p^{\prime}$-DDE ranged from 0.790 to $5.82 \mu \mathrm{g} / \mathrm{kg}$. Much higher concentration of OCPs was found in shrimp's muscle of Lake Taihu than that of Lake Dongting. In addition, the levels of OCPs in shrimps muscle in dry seasons was little higher than in rainy season for two lakes.

Keywords: Gas Chromatography/Mass Spectrometry; shrimp; organochlorine pesticides(OCPs); Lake Taihu; Lake Dongting

有机氯农药 $(\mathrm{OCPs})$ 由于其杀虫效果显著，曾被世界各国广泛使用. 这类农药化学性质稳定、不易降 解和代谢, 具有远距离迁移能力和很高的毒性, 属于典型的持久性有机污染物(POPs). 此外这类农药还 具有高疏水性和脂溶性的特征，极易累积于生物体中，并可通过食物链的传递作用最终危害人体健康， 目前全球都面临着POPs污染问题 ${ }^{[1]}$. 2001年5月由各国政府签署的《关于持久性有机污染物的斯德哥尔摩 公约》(POPs公约)中首批受控的POPs物质中就有 9 种有机氯农药 ${ }^{[2]}$. 由于这些物质对生态系统、环境和人 体健康的负面影响日益凸现, 因而引起各国学者的高度重视和全球社会的普遍关注, 尤其在生物和生态 毒理学方面已成为当前的研究热点和前沿领域 ${ }^{[3]}$.

湖泊被认为是 POPs 的最终汇集地之一, 湖泊水交换周期长, 对污染物的稀释能力较弱, 是一脆弱的 生态系统, 湖泊一旦受到 POPs 污染会遍及整个湖泊生态系统 ${ }^{[4]}$. 湖泊水生生物可通过水体悬浮颗粒物、 沉积物和食物等从它们的生存环境中富集 POP ${ }^{[3]}$, 对水生动物的生长发育构成一定程度的影响, 并有可

\footnotetext{
* 2008-10-28收稿; 2009-03-06收修改稿. 史双昕, 男, 1973年生, 硕士, 工程师; E-mail: shishuangxin2001@163.com.
} 
能通过食物链转移给人类. 处在湖泊食物链顶端食鱼的鱼类和食鱼的水鸟, 它们身体组织和蛋中的POPs 浓度很高, 是较适合监测湖泊POPs污染的“指示生物”[[] , 但是由于这些生物的生命周期一般较长, 加之湖 泊生态环境的变迁, 鱼类和食鱼水鸟作为指示生物不一定能够正确指示反映近期湖泊POPs污染的状况 ${ }^{[6]}$.

青虾在中国是一种分部广泛的淡水虾类, 营养丰富味道极佳, 是人们喜爱的水产品. 青虾在不同年 龄段捕食水体浮游生物和底泥生物, 体内富集了大量POPs, 青虾的生命周期在两年之内, 可作为生物指 示物种反映近期青虾栖息水环境POPs污染的背景水平. 另外, 国外还有学者利用虾类的生物毒性反应来 确定风险评估值来指示环境介质的风险程度 ${ }^{[7-8]}$. 因此研究青虾体内有机氯农药的残留具有科学价值并 对保护居民的身体健康具有重要现实意义.

目前在我国, 研究水环境中 POPs 的介质多集中在沉积物相和水相上 ${ }^{[9-13]}$, 对水生生物的研究近年才 有所增加, 而且大多集中在鱼类和贻贝、牡蚛等少数水生物种上 ${ }^{[14-22]}$. 研究的目标化合物多集中在滴滴 涕和六六六上, 其他种类的持久性有机物研究的相对较少 ${ }^{[16-22]}$. 在国内研究太湖水环境 POPs 残留的较多, 而研究洞庭湖的较少 ${ }^{[1-13,17,20-21]}$, 本文选取太湖和洞庭湖青虾为研究对象, 对 POPs 公约中首批受控的 8 种有机氯农药共 14 种目标化合物进行了分析研究.

\section{1 实验部分}

\section{1 样品采集与预处理}

在太湖 8 个代表湖区和洞庭湖 7 个代表湖区共设采集点 15 个, 太湖 $1^{\#}$ 至 $8^{\#}$ 采样点位为: 东西太湖界点, 沈家, 漫山, 荠嘴, 贡湖, 拖山, 焦山, 大浦(图1), 洞庭湖 $1^{\#}$ 至 $7^{\#}$ 采样点位为: 南岳坡, 君山, 麻塘, 白塘, 营田, 东南湖, 白沙(图2). 2006年4月和9月使用捕虾网分别在各点位采集野生青虾样品29组(白沙点位4月份 未采集到样品), 每组青虾样品采集质量大于 $1 \mathrm{~kg}$, 装人密封的塑料袋置人车载冰箱, 运回实验室分析.

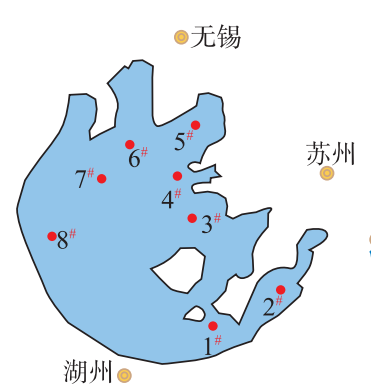

图1 太湖青虾采样点位

Fig.1 Sampling sites of shrimps in Lake Taihu

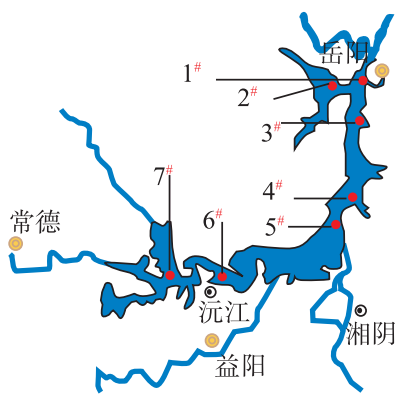

图2 洞庭湖青虾采样点位

Fig.2 Sampling sites of shrimps in Lake Dongting

\section{2 试剂材料与仪器}

1.2.1 化学试剂 实验用丙酮、正已烷、乙酸乙酯试剂(TEDIA公司)均为农残级. 氯化钠、无水硫酸钠(天 津市化学试剂厂): 优级纯, 在 $400^{\circ} \mathrm{C}$ 灼烧 $6 \mathrm{~h}$, 冷却后储于密闭容器中.

氨基SPE小柱(安捷伦公司): 标准型, $1000 \mathrm{mg}(6 \mathrm{ml})$; 高纯氮气: $99.999 \%$, 用于样品浓缩; 高纯氦气: $99.999 \%$, 用于 $\mathrm{GC} / \mathrm{MS}$ 载气.

色谱标准物 $(100 \mu \mathrm{g} / \mathrm{ml}): p, p^{\prime}-\mathrm{DDT} 、 o, p^{\prime}-\mathrm{DDT} 、 p, p^{\prime}$-DDE、 $o, p^{\prime}-\mathrm{DDE} 、 p, p^{\prime}-\mathrm{DDD} 、 o, p^{\prime}-\mathrm{DDD}$ 、七氯、顺 式氯丹、反式氯丹、六氯苯、艾氏剂、狄氏剂、异狄氏剂、灭蚁灵, 购自和光纯药工业株式会社.

回收率指示物 $(100 \mu \mathrm{g} / \mathrm{ml})$ : 滴滴涕 ${ }^{13} \mathrm{C}\left(p, p^{\prime}-\mathrm{DDT}-{ }^{13} \mathrm{C}_{12}\right)$ 购自和光纯药工业株式会社. 内标物 $(100 \mu \mathrm{g} / \mathrm{ml})$ : 氛代菲、氛代萠、氛代屈, 购自和光纯药工业株式会社.

1.2.2 仪器 车载制冷冰箱(北京壹伍陆科技公司); 多功能食品加工机(SQ2119, 上海帅佳电子); 均质仪 (FA25 FLUKO公司); 配自动进样器气相色谱-质谱联用仪(岛津公司QP2010 GC/MS); 旋转蒸发仪(RE111 $\mathrm{BÜCHI}$ 公司)及真空原; 平行蒸发仪(BV180柴田公司); 分液漏斗; 振荡器. 所用器血均经超声波清洗器清 
洗，再经自来水、蒸馏水冲洗，于 $180 \pm 2^{\circ} \mathrm{C}$ 烘箱中烘干，临用前经有机溶剂淋洗.

\section{3 样品处理}

1.3.1 加速溶剂提取仪萃取 将青虾剥去头部和外壳, 只保留青虾的肌肉组织. 取 $100 \mathrm{~g}$ 左右青虾肌肉在食 品加工机上破碎均匀, 准确称取 $5.00 \mathrm{~g}$ 肌肉至 $100 \mathrm{ml}$ 试管中, 加人 $50 \mathrm{ml}$ 丙酮作为萃取试剂, 再加人 $20 \mu \mathrm{l}$ $5 \mu \mathrm{g} / \mathrm{ml}$ 的回收率指示物溶液 $\left(p, p^{\prime}-\mathrm{DDT}-{ }^{13} \mathrm{C}_{12}\right.$ ) 后置于均质仪上, 以 $20000 \mathrm{r} / \mathrm{min}$ 转速均质萃取 $5 \mathrm{~min}$. 将萃取液真 空抽滤, 抽滤液完全转移至 $500 \mathrm{ml}$ 分液漏斗, 加入纯水 $350 \mathrm{ml}$ 和 $17 \mathrm{~g}$ 氯化钠, 充分震荡使氯化钠完全溶解后加 人 $40 \mathrm{ml}$ 正己烷/乙酸乙酯 $=3: 2(\mathrm{~V}: \mathrm{V})$ 的混合溶剂, 震荡 $10 \mathrm{~min}$ 后静置 $10 \mathrm{~min}$ 待其分层, 分离有机相, 水相加人 $40 \mathrm{ml}$ 正己烷和乙酸乙酯的混合溶剂重复以上步骤, 分液后的有机相与第一次分离的有机相合并, 合并溶液转 移到茄形瓶中后在旋转蒸发器上浓缩至 $10 \mathrm{ml}$, 加 $3-5 \mathrm{~g}$ 无水硫酸钠脱水过滤, 滤液转移至 $200 \mathrm{ml}$ 茄形瓶中在旋 转蒸发器上浓缩至约 $2 \mathrm{ml}$ 后, 向茄形瓶中加人 $15 \mathrm{ml}$ 正己烷, 在旋转蒸发器上浓缩至约 $2 \mathrm{ml}$, 连续重复该步骤 2 次 用来替换溶剂. 将最终的浓缩液完全转移至 $10 \mathrm{ml}$ 具塞刻度试管中, 用平行蒸发仪浓缩至约 $1 \mathrm{ml}$, 待净化.

1.3.2 净化分离步骤 将氨基SPE小柱 $(1000 \mathrm{mg})$ 用 $18 \mathrm{ml}$ 正己烷活化. 将待净化的样品溶液转移至已活化的 氨基SPE小柱上, 加人 $13 \mathrm{ml}$ 正己烷洗脱, 用 $20 \mathrm{ml}$ 具塞刻度试管接收全部洗脱液. 用平行蒸发仪浓缩至约 $1 \mathrm{ml}$. 加人 $20 \mu 1$ 内标溶液 $(5 \mu \mathrm{g} / \mathrm{ml})$, 用正己烷定容至 $1.0 \mathrm{ml}$ 后, 备 $\mathrm{GC} / \mathrm{MS}$ 分析用, 进样量 $2 \mu 1$.

\section{4 有机氯农药类POPs的GC/MS分析}

色谱柱: DB-1 $30 \mathrm{~m} \times 0.32 \mathrm{~mm}$ i.d. $\times 0.25 \mu \mathrm{m} \mathrm{df}$; 进样口温度: $250^{\circ} \mathrm{C}$; 色谱-质谱接口温度: $270^{\circ} \mathrm{C}$; 离子 源温度： $230^{\circ} \mathrm{C}$; 进样方式：不分流进样; 程序升温条件： $70^{\circ} \mathrm{C}$ (保持 $\left.1 \mathrm{~min}\right)$, 以 $20^{\circ} \mathrm{C} / \mathrm{min}$ 升至 $130^{\circ} \mathrm{C}$, 再以 $5^{\circ} \mathrm{C} / \min$ 升至 $210^{\circ} \mathrm{C}$, 最后以 $15^{\circ} \mathrm{C} / \mathrm{min}$ 升至 $300^{\circ} \mathrm{C}$.

离子源： $\mathrm{EI} 70 \mathrm{eV}$; 定性分析：以全扫描方式(SCAN), 扫描范围 $(m / z)$ 为 $35-500$; 定量分析：选择离子 检测 $(\mathrm{SIM})$ 方式. 每个工作日在分析之前，选用 $5 \mu \mathrm{g} / \mathrm{ml} p, p^{\prime}$-DDT和 $o, p^{\prime}-\mathrm{DDT}$ 的混合标准溶液，进样 $1 \mu 1$, 以 全扫描方式测定，检查GC进样口是否引起DDT的降解. 只有DDT分解为DDD和DDE的程度小于 $15 \%$ 仪 器方能用于样品的测定.

\section{2 质量控制及保证}

\section{1 仪器检出限与方法检出限}

使用5点校正内标法定量, 标准曲线的浓度为 $5 、 10 、 50 、 100 、 250 \mathrm{ng} / \mathrm{ml}$, 对浓度为 $5 \mathrm{ng} / \mathrm{ml}$ 标准溶液 平行分析6次, 取其标准偏差 $s$, 仪器检出限为 $3 s$, 定量下限为 $10 s($ 表 1 ).

表1 仪器检出限及定量限 $(\mathrm{ng} / \mathrm{ml})$

Tab.1 The instrument detection limit and quantitative limit

\begin{tabular}{llllll}
\hline 化合物名称 & 浓度平均值 & $\mathrm{RSD}(\%)$ & $s$ & $3 s$ & $10 s$ \\
\hline 六氯苯 & 4.93 & 0.85 & 0.0807 & 0.242 & 0.807 \\
七氯 & 5.02 & 2.25 & 0.217 & 0.651 & 2.17 \\
艾氏剂 & 4.93 & 0.77 & 0.0727 & 0.218 & 0.727 \\
反式氯丹 & 5.15 & 2.94 & 0.291 & 0.872 & 2.91 \\
$o, p^{\prime}-\mathrm{DDE}$ & 5.03 & 1.84 & 0.178 & 0.534 & 1.78 \\
顺式氯丹 & 5.03 & 3.29 & 0.318 & 0.955 & 3.18 \\
$p, p^{\prime}-\mathrm{DDE}$ & 4.98 & 0.95 & 0.0909 & 0.273 & 0.909 \\
狄氏剂 & 5.40 & 2.86 & 0.297 & 0.890 & 2.97 \\
$o, p^{\prime}-\mathrm{DDD}$ & 5.10 & 1.31 & 0.129 & 0.386 & 1.29 \\
异狄氏剂 & 5.18 & 3.57 & 0.355 & 1.07 & 3.55 \\
$p, p^{\prime}-\mathrm{DDD}$ & 4.90 & 1.76 & 0.166 & 0.498 & 1.66 \\
$o, p^{\prime}-\mathrm{DDT}$ & 4.83 & 1.53 & 0.142 & 0.427 & 1.42 \\
$p, p^{\prime}-\mathrm{DDT}$ & 4.80 & 1.83 & 0.0169 & 0.508 & 1.69 \\
灭蚁灵 & 4.97 & 3.78 & 0.173 & 0.518 & 1.73 \\
\hline
\end{tabular}


方法可靠性和重复性采用基体加标回收试验验证，14种目标化合物回收率为 $87.1 \%-121 \%$ (表2). 对实 际样品使用 Surrogates $\left(p, p^{\prime}\right.$-DDT- $\left.{ }^{13} \mathrm{C}_{12}\right)$ 监控前处理过程. 每 10 个样品或每批样品分析时增加全分析过程空 白, 并且进行一个样品的重复测定.

表2 加标回收率(\%)和重复性试验

Tab.2 Standard addition recovery test and injection repeatability test

\begin{tabular}{llllllll}
\hline 化合物名称 & 1 & 2 & 3 & 4 & 5 & 平均值 & $\mathrm{RSD}(\%)$ \\
\hline 六氯苯 & 86.7 & 89.9 & 85.7 & 80.7 & 93.4 & 87.3 & 5.45 \\
七氯 & 76.4 & 85.0 & 100 & 94.8 & 88.6 & 88.9 & 10.2 \\
艾氏剂 & 85.2 & 89.0 & 86.2 & 82.1 & 92.9 & 87.1 & 4.69 \\
反式氯丹 & 86.2 & 87.3 & 89.6 & 85.7 & 90.4 & 87.8 & 2.36 \\
$o, p^{\prime}-\mathrm{DDE}$ & 86.1 & 85.2 & 106 & 86.2 & 80.1 & 88.8 & 7.02 \\
顺式氯丹 & 85.7 & 87.4 & 85.8 & 85.1 & 89.9 & 86.8 & 2.24 \\
$p, p^{\prime}$-DDE & 111 & 111 & 118 & 110 & 114 & 113 & 2.24 \\
狄氏剂 & 96.1 & 96.8 & 96.5 & 91.3 & 95.7 & 95.3 & 2.36 \\
$o, p^{\prime}$-DDD & 86.9 & 108 & 84.1 & 84.4 & 79.8 & 88.6 & 7.72 \\
异狄氏剂 & 120 & 124 & 124 & 116 & 119 & 121 & 2.38 \\
$p, p^{\prime}-\mathrm{DDD}$ & 99.7 & 99.8 & 107 & 97.8 & 100 & 101 & 2.90 \\
$o, p^{\prime}-\mathrm{DDT}$ & 94.1 & 101 & 105 & 99.9 & 100 & 100 & 2.85 \\
$p, p^{\prime}$-DDT- ${ }^{13} \mathrm{C}_{12}$ & 119 & 130 & 118 & 130 & 113 & 122 & 3.51 \\
$p, p^{\prime}$-DDT & 81.7 & 97.4 & 92.9 & 98.7 & 90.9 & 92.3 & 3.90 \\
灭蚁灵 & 97.7 & 102 & 108 & 101 & 105 & 102 & 6.27 \\
\hline
\end{tabular}

\section{3 结果与讨论}

\section{1 太湖洞庭湖青虾肌肉中有机氯农药含量}

所有青虾样品中氯丹、艾氏剂、狄氏剂、异狄氏剂、七氯和灭蚁灵均未检出，只有六氯苯、 $p, p^{\prime}-\mathrm{DDE}$ 和 $p, p^{\prime}-\mathrm{DDD}$ 有检出, 六氯苯的检出率为 $75.9 \%, p, p^{\prime}$-DDE的检出率为 $100 \%$, 洞庭湖的南岳坡点位的青虾肌 肉中检测出了 $p, p^{\prime}-\mathrm{DDD}$ (表 3 , 表4). 太湖青虾肌肉中六氯苯和 $\sum D D T s(D D T+D D E+D D D)$ 平均含量为 0.331 和 $1.61 \mu \mathrm{g} / \mathrm{kg}$, 洞庭湖青虾的含量为 1.58 和 $2.16 \mu \mathrm{g} / \mathrm{kg}$. 太湖青虾肌肉中六氯苯在枯水期和丰水期时的平均 含量分别为 0.369 和 $0.294 \mu \mathrm{g} / \mathrm{kg}, p, p^{\prime}$-DDE在枯水期和丰水期时的含量平均分别为 1.49 和 $1.74 \mu \mathrm{g} / \mathrm{kg}$. 洞庭湖 青虾肌肉中六氯苯在枯水期和丰水期时的平均含量分别为 3.22 和 $0.173 \mu \mathrm{g} / \mathrm{kg}, \sum \mathrm{DDTs}$ 在枯水期和丰水期时 的平均含量分别为 4.15 和 $2.75 \mu \mathrm{g} / \mathrm{kg}$.

表3 太湖青虾肌肉中六氯苯和滴滴涕的含量 $(\mu \mathrm{g} / \mathrm{kg}(\text { 湿重 }))^{*}$

Tab.3 HCB and $\sum$ DDTs levels in shrimps of Lake Taihu

\begin{tabular}{|c|c|c|c|c|c|c|c|c|c|c|c|c|c|c|c|c|}
\hline \multirow{2}{*}{ OCPs } & \multicolumn{8}{|c|}{ 枯水期 } & \multicolumn{8}{|c|}{ 丰水期 } \\
\hline & $1^{\#}$ & $2^{\#}$ & $3^{\#}$ & $4^{\#}$ & $5^{\#}$ & $6^{\#}$ & $7^{\#}$ & $8^{\#}$ & $1^{\#}$ & $2^{\#}$ & $3^{\#}$ & $4^{\#}$ & $5^{\#}$ & $6^{\#}$ & $7^{\#}$ & $8^{\#}$ \\
\hline $\mathrm{HCB}$ & nd & nd & 0.210 & 0.391 & 0.264 & 1.24 & 0.315 & 0.532 & nd & nd & 0.214 & nd & 0.175 & 1.19 & 0.257 & 0.513 \\
\hline$p, p^{\prime}-\mathrm{DDE}$ & 0.940 & 2.12 & 1.48 & 1.64 & 1.12 & 2.03 & 1.37 & 1.21 & 2.00 & 2.17 & 1.29 & 2.12 & 1.97 & 1.88 & 1.67 & 0.790 \\
\hline
\end{tabular}

\section{2 太湖洞庭湖青虾肌肉中有机氯农药残留特征及初步研究}

青虾肌肉中艾氏剂、狄氏剂、异狄氏剂和灭蚁灵未检出，这与历史上两湖流域未用或很少使用过这 些杀虫剂有关. 六氯苯和 $\sum$ DDTs则是野生青虾肌肉中广泛存在的两种有机氯农药, 这与两个湖水环境中 六氯苯和滴滴涕的残留历史也相符, 资料显示, 多年来六氯苯和滴滴涕在两湖流域广泛使用, 最终残留 
在湖泊水环境中 ${ }^{[10-13,23]}$. 总体上太湖的青虾肌肉中检测出的有机氯农药含量比洞庭湖青虾肌肉中的低.

青虾肌肉的滴滴涕含量不高. 参照GB2763-2005食品中农药最大残留限量, 水产品滴滴涕限量为 $500 \mu \mathrm{g} / \mathrm{kg}$ (湿重)，两个湖青虾肌肉中的 $\sum \mathrm{DDT}$ 都未超标. 原因之一是青虾肌肉组织比消化腺和脂肪组织 富集OCPs的能力低, 水生生物体内脂含量越高, 其OCPs 含量也越高, $\sum$ DDTs浓度也会增加 ${ }^{[14,24-25]}$. 原因 二是青虾处于食物链的底层, 主要经被动扩散由身体表面直接从水和底泥中吸收 $\mathrm{OCPS}^{[26]}$, 加之青虾寿命 短新陈代谢低, 富集OCPs能力比食物链上层的肉食性生物差, 因此青虾肌肉中的OCPs含量较低 ${ }^{[22]}$.

表4 洞庭湖青虾肌肉中六氯苯和滴滴涕的含量 $(\mu \mathrm{g} / \mathrm{kg}(\text { 湿重 }))^{*}$

Tab.4 HCB and $\sum$ DDTs levels in shrimps of Lake Dongting

\begin{tabular}{|c|c|c|c|c|c|c|c|c|c|c|c|c|c|}
\hline \multirow{2}{*}{ OCPs } & \multicolumn{6}{|c|}{ 枯水期 } & \multicolumn{7}{|c|}{ 丰水期 } \\
\hline & $1^{\#}$ & $2^{\#}$ & $3^{\#}$ & $4^{\#}$ & $5^{\#}$ & $6^{\#}$ & $1^{\#}$ & $2^{\#}$ & $3^{\#}$ & $4^{\#}$ & $5^{\#}$ & $6^{\#}$ & $7^{\#}$ \\
\hline $\mathrm{HCB}$ & 0.589 & 1.12 & 1.10 & 0.890 & 13.2 & 2.43 & 0.416 & nd & 0.287 & 0.289 & nd & 0.220 & nd \\
\hline$p, p^{\prime}-\mathrm{DDE}$ & 2.13 & 4.10 & 4.30 & 3.00 & 3.21 & 3.99 & 3.10 & 1.00 & 5.82 & 2.28 & 1.95 & 2.31 & 2.77 \\
\hline$p, p^{\prime}-\mathrm{DDD}$ & 0.599 & nd & nd & nd & nd & nd & nd & nd & nd & nd & nd & nd & nd \\
\hline
\end{tabular}

*nd: 未检出.

两湖青虾肌肉中检出的 $\sum$ DDTs主要是 $p, p^{\prime}-\mathrm{DDE}$, 其中一部分是青虾富集水环境中本身残留的 DDE(包 括o, $p^{\prime}$-DDE和 $\left.p, p^{\prime}-\mathrm{DDE}\right)$, 这些DDE主要是长期以来地表径流、大气沉降和工业污水排放等人湖滴滴涕降解 产生的 ${ }^{[13]}$. 另外一部分是青虾代谢自身富集DDT(包括o, $p^{\prime}$-DDT和 $p, p^{\prime}$-DDT)产生的DDE，这是由于DDT进 人生物体后异构体很快发生变化, $p, p^{\prime}-\mathrm{DDT}$ 转化为 $p, p^{\prime}-\mathrm{DDD}$ 和 $p, p^{\prime}-\mathrm{DDE}^{[27]}$. 对 $\sum \mathrm{DDTs}$ 的不同同系物,不同生 物和同种生物不同组织或器官, DDT积累和代谢特征不同, 有的以DDE为主, 有的以DDD为主 ${ }^{[28-29]}$. 本研究 说明青虾肌肉中残留和代谢DDT的产物主要为DDE. 有研究证明, 水生生物体中高水平的 $p, p^{\prime}$-DDT是水环 境中有新的DDT污染源输人的重要特征. 另外, DDD/DDE以及(DDD+DDE)/DDT 这两个比值也常用来示 踪DDT降解程度, 并被用于判定有无新滴滴涕的输人 ${ }^{[30-31]}$. 由于本研究只对青虾的肌肉组织进行了检测, 而肌肉中的滴滴涕含量较低, 要判断是否有新的滴滴涕输人两湖, 需要进一步研究.

两个湖青虾肌肉中的六氯苯和 $\sum$ DDTs 在枯水期的含量多数大于丰水期的含量. 原因一是丰水期的越 冬成虾比例少, 而幼虾主要以藻类、水草茎叶碎片、浮游动物、细菌、泥沙等为食, 成年青虾则主要捕

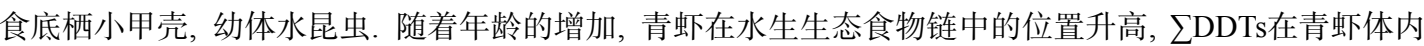
的含量也逐渐升高，因此枯水期的成虾富集的 $\sum$ DDTs含量更多一些. 另外，丰水期的大量来水对两湖水 环境中农药的稀释作用也不可忽视.

六氯苯是野生青虾肌肉中广泛存在的一种OCPs, 六氯苯的来源很复杂, 湖泊沉积物中的六氯苯主要 来源为地表径流人湖、大气沉降和工业污水排放 ${ }^{[10,32]}$. 另外一个重要的途径是由于生产和使用五氯酚和 五氯酚钠造成的 ${ }^{[33-34]}$. 五氯酚和五氯酚钠曾作为我国血吸虫病疫区常用的杀灭血吸虫中间宿主钉螺的药 物, 在两湖长时间施用, 在使用五氯酚及五氯酚钠的过程中会向两湖水环境中释放六氯苯 ${ }^{[33]}$. 太湖和洞 庭湖青虾肌肉中的六氯苯来源是由多种因素造成的.

致谢：感谢联合国大学和岛津公司的指导, 太湖渔业管理委员会的采样协助.

\section{4 参考文献}

[1] 黄 俊, 余 刚, 钱 易. 我国的持久性有机污染物问题与研究对策. 环境保护, 2001, 11: 3-6.

[2] 联合国环境规划署. 关于对某些持久性有机污染物的斯德哥尔摩公约. 斯德哥尔摩: 联合国环境规划署, 2001.

[3] 李雪梅, 张庆华, 甘一萍等. 持久性有机污染物在食物链中积累与放大研究进展. 应用与环境生物学报, 2007, 13(6): 901-905.

[4] Tsuda T, Nakajima A, Kojima M et al. Polychlorinated biphenyls and organochlorine insecticides residues in fish and Shellfish from Lake Biwa. Toxicological Environmental Chemistry, 1999, 71(3-4): 309 -317. 
[5] 董元华, 安 琼, 龚钟明等. 太湖湿地生态系统有机氯污染的夜鹭生物指示. 应用生态学报, 2002, 13(2): 209-212.

[6] Hebert CE, Hobson KA, Shutt JL. Changes in food web structure affect rate of PCB decline in herring gull (Larus argentatus) eggs. Environ Sci \& Technol, 2000, 34(9): 1609-1614.

[7] Long ER, MacDonald DD, Smith SL et al. Incidence of adverse biological effects with ranges of chemical concentrations in marine and estuarine sediments. Environmental Management, 1995, 19(1): 81-97.

[8] Long ED, Field LJ, Macdonald DD. Predicting toxicity marine sediments with numerical sediment quality guidelines. Environ Toxicol Chem, 1998, 17(4): 714-727.

[9] 李国刚, 李红莉. 持久性有机污染物在中国的环境监测现状. 中国环境监测, 2004, 20(4): 53-60.

[10] 袁旭音, 王 禹, 陈 骏等. 太湖沉积物中有机氯农药的残留特征及风险评估. 环境科学, 2003, 24(1): 121-125.

[11] 李炳华, 任仲宇, 陈鸿汉等. 太湖流域某农业区浅层地下水有机氯农药残留特征初探. 农业环境科学学报, 2007, 26(5): 1714-1718

[12] 乔 敏, 王春霞, 黄圣彪等. 太湖梅梁湾沉积物中有机氯农药的残留现状. 中国环境科学, 2004, 24(5): 592-595.

[13] 刘国卿, 林海涛, 张 干等. 太湖沉积物中有机氯农药的污染历史. 中国环境科学, 2007, 27(4): 441-444.

[14] 丘耀文, 张 干, 郭玲利等. 大亚湾海域典型有机氯农药生物累积特征及变化因素研究. 海洋学报, 2007, 29(2): 51-58.

[15] 周明莹, 乔向英, 崔 毅等. 青岛沿海养殖区贝类体内有机氯农药残留量分布和评价. 海洋环境科学, 2008, 27(1): 6-9.

[16] 李 荣, 甘金华, 徐 进等. 长江宜昌江段铜鱼和中华鲟体内 HCH DDT 的残留水平. 农业环境科学学报, 2008, 27(6): 2434-2439.

[17] 赵 肖, 张娅兰, 李适宇. 滴滴涕对太湖经济鱼类危害的生态风险. 生态学杂志, 2008, 27(2): 295-299.

[18] 郭建阳，孟祥周，麦碧娴等. 滴滴涕类农药在广东省沿海区水产品中的残留及人类暴露水平. 生态毒理学报，2006，1(3): $236-242$.

[19] 董 军, 奕天罡, 邹世春等. 珠江三角洲淡水养殖沉积物及鱼体中 DDTs 和 PAHs 的残留与风险分析. 生态环境, 2006, 15(4): 693-696

[20] 边学森, 刘洪波, 甘居利等. 太湖五里湖背角无齿蚌体内滴滴涕和六六六的残留. 生态与农村环境学报, 2007, 23(2): 52-56.

[21] 李天云, 黄圣彪, 孙 凡. 河蚬对太湖梅梁湾沉积物中 HCHs 和 DDTs 的生物富集. 环境工程学报, 2008, 2(8): 1009-1006.

[22] 骆世昌, 余汉生. 珠江口及附近海域生物体中 BHC 和 DDT 的含量研究. 海洋通报, 2001, 20(2): 44-50.

[23] 赵中华, 张 路, 于 釒等. 太湖表层沉积物中有机氯农药残留及遗传毒性初步研究. 湖泊科学, 2008, 20(5): 579-584.

[24] Kidd KA, Bootsma HA, Hesslein RH et al. Biomagnification of DDT through the benthic and pelagic food webs of Lake Malawi, East Africa: Importance of trophic level and carbon source. Environ Sci \& Technol, 2001, 35(1): 14-20.

[25] Kumblad L, Olsson A, Koutny V et al. Distribution of DDT residues in fish from the Songkhla Lake, Thailand. Environmental Pollution, 2001, 112(2): 193-200

[26] Naso B, Perrone D, Ferrante MC et al. Persistent organic pollutants in edible marine species from the Gulf of Naples, Southern Italy. Sci Total Environ, 2005, 343(1-3): 83-95.

[27] Suns K, Hitchin G, Adamek E. Present status and temporal trends of organochlorine contaminants in Young-of-the-year Spottail shiners (Notropis hudsonius) from Lake Ontario, Canada. Canada Journal of Fish Aquatic Science, 1991, 48(8): 1568-1573.

[28] Leland JJ, Atephen RC, Jonmn et al. PCB congeners in Lake Michigan coho (Oncorhynchus Kisutch ) and Chinook (Oncorhynchus Tshawytscha ) salmon. Environ Sci Technol, 2001, 35(5): 856-862.

[29] Sapozhnikova Y, Bawardi O. Schlenkd pesticides and PCBs in sediments and fish from the Salton Sea, California, USA Chemosphere, 2004, 55(6): 797-809.

[30] Kosatsky T, Przybysz R, Shatenstein et al. Fish consumption and contaminant expose among Montreal-Area sportfishers pilot study. Environ Res, 1999, 80(2): 150-158.

[31] Hitch RK, Day HP. Unusual persistence of DDT in some Western USA soils. Bull Environ Contam Toxicol, 1992, 48(2): $255-264$.

[32] 吴荣芳, 解清杰, 黄卫红等. 六氯苯的环境危害及其污染控制. 化学与生物工程, 2006, 23(8): 7-10.

[33] 张 兵, 郑明辉, 刘芃岩等. 五氯酚在洞庭湖环境介质中的分布. 中国环境科学, 2001, 21(2): 165-167.

[34] 王 俊, 孙津生. 河流底泥沉积物中五氯酚和六氯苯残留的同时测定. 质谱学报, 2006, 27(2): 79-89. 